\title{
EVALUATION OF DIAGNOSTIC UTILITY OF USING TWO SUBSTRATES FOR ENDOMYSIUM ANTIBODIES TEST WITH COMPARISON WITH HISTOLOGICAL FINDINGS IN IRAQI COELIAC PATIENTS
}

\author{
MUHAMED T. OSMAN ${ }^{* 1}$, GHADA AL-DUBONI², SANA'A A AL-NASIRY ${ }^{3}$, MAKKI H. F. ${ }^{4}$ AND BALSAM I. T. ${ }^{5}$ \\ 1Department of Pathology, Faculty of Medicine, Universiti Teknologi MARA UiTM Malaysia. \\ 2Department of Basic Sciences, College of Dentistry, University of Baghdad, Baghdad, Iraq. \\ ${ }^{3}$ Department of Pathology, College of Medicine, University of Baghdad. Baghdad, Iraq. \\ 4FRCP, Consultant Gastroenterologist, GIT Hospital, Medical City, Baghdad, Iraq. \\ ${ }^{5} \mathrm{DCP}$, Specialized Surgeries Hospital, Medical City, Baghdad, Iraq. \\ ${ }^{*}$ Corresponding Author: Email- mtosman2004@gmail.com
}

Received: February 24, 2012; Accepted: March 06, 2012

\begin{abstract}
-
Purpose: A case-control study was carried out to elucidate prospectively the sensitivity and specificity of endomysium antibodies by using two substrates in the diagnosis of coeliac disease as a first attempt in an Iraqi population with comparison with histological findings.

Methods: The study consisted of 314 patients and 100 normal controls. Oesophago-gastroduodenoscopy was done to each patient and three biopsies from distal duodenum were taken. Venous blood sample was also obtained from each patient and normal subjects. The sera were tested for endomysium antibodies (EMA) by using two substrates; monkey esophagus and human umbilical cord by indirect immunofluorescent technique (IIF).

Results: The sensitivity of EMA (monkey oesophagus) test among both children and adults was $93.8 \%$ and $100 \%$ respectively, while the sensitivity of EMA (umbilical cord) test among children and adults was $96.6 \%$ and $100 \%$ respectively.

Conclusions: The human umbilical cord tissue is better for using as a substrate than the monkey esophagus tissue among Iraqi ceoliac patients. Therefore, the test is suitable as a diagnostic and screening tool for the disease.
\end{abstract}

Key words- Coeliac disease, Endomysium antibody , Indirect immunofluorescent technique, Monkey esophagus, Human umbilical cord.

Citation: Muhamed T. Osman, et al (2012) Evaluation of Diagnostic Utility of Using Two Substrates for Endomysium Antibodies Test with Comparison with Histological Findings in Iraqi Coeliac Patients. International Journal of Medical and Clinical Research, ISSN:0976-5530 \& E -ISSN:0976-5549, Volume 3, Issue 3, pp.-127-131.

Copyright: Copyright(02012 Muhamed T. Osman, et al. This is an open-access article distributed under the terms of the Creative Commons Attribution License, which permits unrestricted use, distribution and reproduction in any medium, provided the original author and source are credited.

\section{Introduction}

Coeliac Disease (CD), also known as gluten-sensitive enteropathy, was traditionally considered an intestinal disease occurs in both children and adults, characterized by malabsorption, diarrhoea, weight loss, abnormal stools and abdominal distention. [1] However more than half of CD patients do not exhibit these "typical" symptoms. The vast array of symptoms that may indicate CD include dermatitis herpetiformis, psychiatric illness, short stature, infertility, malignancy of the gastrointestinal tract, recurrent abdominal pain, indigestion, anemia, macrocytosis and folate deficiency. [2, 3]

The mucosal lesion develops in genetically susceptible individuals after ingestion of dietary gluten and recovers when glutencontaining cereals, wheat, rye and barley are withdrawn from the diet. [4] The disease should be detected as early as possible, because untreated $C D$ is associated with many severe complications such as intestinal lymphoma or cancer and osteoporosis.[4] Serological tests developed in the last two decades to simplify and shorten the time for the diagnosis of $C D$ providing a non-invasive tool to screen both individuals at risk for the disease and the general population. $[3,5]$ However, the current gold standard for the diagnosis of coeliac disease remains histological confirmation of the intestinal damage in serologically positive individuals. [6] Measurement of $\lg A$ antibodies recognizing endomysium by indirect immunofluorescence has historically been considered the gold standard serologic assay for CD. These autoantibodies recognize an intermyofibril substance found in primate smooth muscle connective tissue. $[7,8]$ The tissue of choice for detection of 
endomysial antibodies (EMA) is monkey esophogus; however, many studies indicate that human umbilical cord is also a suitable substrate. Several studies have shown that the sensitivity and specificity of IgA endomysial antibodies for CD using both substrates are both greater than $95 \%$. It is now known that the target autoantigen contained within the endomysium is the enzyme tissue transglutaminase (tTG). [7 - 10]

The purpose of this study was to elucidate prospectively the sensitivity and specificity of EMA by using two substrates in the diagnosis of CD as a first attempt in an Iraqi population with comparison with histological findings.

\section{Subjects, Materials and Methods}

A total of 414 subjects were studied in the Medical city hospitals in Baghdad. Subjects recruited in this case-control study were classified into 2 main groups.

\section{Patient study group}

This group consisted of 314 patients attending Gastro- intestinal teaching hospital, Al Mansour pediatric hospital and Baghdad teaching hospital. These patients were referred from different hospitals in Baghdad and other states in Iraq, because they were suspected on clinical basis to have coeliac disease. The age of those patients ranged from (1) year to (72) years, with an average age of (15) years.

All patients were subjected to a personal interview using especially designed questionnaire format. Oesophago-gastro- duodenoscopy (OGD) was done to each patient and three biopsies from distal duodenum were taken. Venous blood samples were also obtained from each patient and sera subjected to IgA endomysium antibody (EMA) tests.

\section{Control study group}

The control group consisted of 100 apparently healthy individuals (50 adults \& 50 children) who were not complaining of any gastrointestinal problem. Venous blood samples were taken and sera tested for IgA EMA antibodies tests.

$A$ ready kit of EMA by using monkey esophagus substrate (supplied from Medic Company, Italy 1X8: tests) was used. Further more, frozen sections of human umbilical cord was prepared in the histology laboratory of Specialized Surgeries Hospital were used.

All sera from patient study group and control study group subjected to the test using indirect immunofluorescent (IIF) method.

Negative sera, for IgA EMA in highly suspected coeliac patients were subjected to the test with IgG monoclonal conjugate by IIF to exclude IgA deficiency disease associated with coeliac disease.

Sections of the duodenal mucosa were cut at a thickness of 5 micrometer and stained with haematoxylin and eosin (H\&E). Slides were interpreted by two pathologists who were not informed about the clinical status of the patients and interpreted small intestinal histological features, according to the Marsh classification and modified Marsh criteria: [11,12] Marsh I consists of raised intraepithelial lymphocytes (IELs) with >40 lymphocytes per 100 enterocytes, Marsh II consists of raised intraepithelial lymphocytes and crypt hyperplasia, Marsh Illa partial villous atrophy, Marsh IIlb subtotal villous atrophy and Marsh IIlc total villous atrophy. Diagnosis of coeliac disease was dependant on the presence of Marsh III only. Any report, which did not include the features of Marsh III was considered as non-coeliac patient.
All adult subjects and children's parents gave informed written consent and the study was approved by the Ethics Committee of College of Medicine, University of Baghdad, Iraq.

\section{Summery of serologic methods}

By using monkey esophagus sections, as substrate EMA; was considered positive if it corresponds with the pattern described by Chorzelski TP, et al 1984 [9] in coeliac disease. The tests were performed as follows: 5 micrometer sections of fresh frozen monkey oesophagus were air-dried for $20 \mathrm{~min}$. Five microlitres of undiluted serum or of twofold dilutions with an initial dilution of $1: 2.5$ were applied on the sections and incubated for $30 \mathrm{~min}$. The slides were washed in phosphate-buffered saline PBS (From: Sanofi Diagnostic Pasteur., France) for two 10-min periods, followed by 30 min of incubation with Fluorescein labeled anti-human liquid globulin (F.I.T.C.) conjugate (Sanofi diagnostic pasteur kallestedchaska, France) The conjugate characteristics were as follows: F/ $P$ ratio, 2.3; antibody concentration, $100 \mathrm{pg} / \mathrm{ml}$; protein, $0.8 \mathrm{~g} / \mathrm{l}$; and working dilution, 1:64. After being washed twice in PBS the samples were mounted in buffered glycerin. The slides were examined in a Fluorescence microscope (orthanol-Leitiz laborotux, D-Letiz, Wetzler; Germany) with an exciting primary filter K 500 and supplied with a fully automatic camera. IgA specific for the endomysial lining of myofibrils were identified by their reticulin like staining of smooth muscle bundles. Sera samples containing antibodies at any titer were considered positive.

By using human umbilical cord sections as a substrate of EMA; was considered positive if it corresponds with the pattern described by Ladinser B et al, 1994 [10] in coeliac disease. Tests for Briefly, the cryostatic tissue sections were mounted and fixed on microscope slides. Serum samples diluted $1 / 2.5,1 / 5$ and $1 / 10$ with phosphate-buffered saline (PBS) were applied to the slides which were incubated for 30 minutes at room temperature. This initial dilution was chosen because no residual staining was seen at this or higher titres. After washing three times with PBS for 5 minutes, the sections were covered with 1:80 fluorescein-conjugated goat anti-human $\lg A$ for 30 minutes, washed again with PBS, mounted in alkaline glycerin buffer and examined by Fluorescence microscope. Sera from coeliac patients showed a honey comb like fluorescence along the peritubular muscle layers of vessels on human umbilical cord, the typical positive reaction stained the extracellular connective tissue.

\section{Statistical Analysis}

Statistical analyses were done using SPSS v10 for Windows. Frequency distributions were done first and then cross- tabulation for selected variables and the statistical significance of associations was tested with the chi- square test for homogeneity. Multiple logistic regression analysis was employed to study the independent effect of each variable included in the model, after controlling for the others, on the probability of having a positive EMA tests. The performance characteristics of a test, sometimes called test operating characteristics include, among others: sensitivity specificity, positive predictive value and negative predictive value. Data values were adjusted for age and initial values. Analyses where the $P$-value was $<0.05$ were considered to be statistically significant. 


\section{Results}

The results presented in this study were based on the analysis of data on a total of 314 subjects in whom CD was suspected on clinical grounds and 100 normal controls. Since children with CD differ from adults in certain aspects, the associations presented in this study grouped into two categories; first, children ( $<18$ years) (female to male ratio 1.29:1) second, adults ( $\geq 18$ years) (female to male ratio 0.82:1). Duodenal biopsies revealed histopathological changes of CD (Marsh III; villous atrophy) in 226 cases from 314 patients, (155 children and 71 adults). The remaining 88 were labeled as non-coeliac patients.

EMA (mo): EMA by using monkey oesophagus substrate: As shown in table 1, there was a statistically significant positive trend between EMA (mo) positivity rate and the severity of histological changes in coeliac patients. The rate increased from $4.5 \%$ for patients with Marsh I (partial villous atrophy) to $98 \%$ for those with Marsh IIlc (total villous atrophy ), figure 1.

Among children, a much high proportion $87.7 \%$ of patients with CD showed positive test for EMA (mo) compared to $2.1 \%$ and $0 \%$ for non-coeliac and control groups respectively and this difference was highly significant statistically. The same was applicable for adults where the EMA (mo) positivity rate ranged from $100 \%$ in the coeliac group to $0 \%$ in both non-coeliac and control groups.

The differences in EMA (mo) positivity rates between both gender groups were small in children and non in adults, while the EMA (mo) positivity rate was $87.7 \%$ in children with CD compared to adults $100 \%$. As shown in table 2, the PPV of EMA (mo) test in both children and adults was very high, ranging from $99.3 \%$ to $100 \%$ for patients with a clinical suspicion of coeliac disease, also the NPV was high ranging from $71.21 \%$ to $100 \%$ in children and adults respectively. The sensitivity of EMA (mo) test among children was $87.72 \%$ while it was $100 \%$ among adults. If this test used to be in screening for coeliac disease one can expect to find $87.72 \%$ of children and $100 \%$ of adults who showed positivity are real coeliac patients, table 2 .

Table 1- EMA (mo) positivity rates according to histopathological findings

\begin{tabular}{|lllll|}
\hline \multirow{2}{*}{ Histopathology } & \multicolumn{2}{l}{ Positive EMA(mo) } & Total & P-value \\
\hline Marsh I & No. & $\%$ & No. & \\
Marsh II & 0 & 0 & 29 & \\
Marsh IIla & 1 & 4.5 & 22 & \\
Marsh IIIb & 27 & 65.9 & 41 & \multirow{2}{*}{0.00001} \\
Marsh IIIc & 84 & 96.6 & 87 & \\
Normal histology & 96 & 98 & 98 & \\
Total & 0 & 0 & 37 & \\
\hline
\end{tabular}

Table 2- Performance characteristics of EMA (mo) test (Sensitivity and Specificity)

\begin{tabular}{|llll|}
\hline Children $(\mathrm{n}=202)$ & EMA (mo) test & Coeliac & Non-coeliac \\
Sensitivity $=87.72 \%$ & Positive & 136 & 1 \\
Specificity $=97.87 \%$ & Negative & 19 & 46 \\
PPV $=99.3 \%$ & Total & 155 & 47 \\
NPV $=71.21 \%$ & & & \\
Adults $(n=112)$ & EMA (mo) test & Coeliac & Non-coeliac \\
Sensitivity $=100 \%$ & Positive & 71 & 0 \\
Specificity $=100 \%$ & Negative & 0 & 41 \\
PPV $=100 \%$ & Total & 71 & 41 \\
NPV $=100 \%$ & & & \\
\hline
\end{tabular}

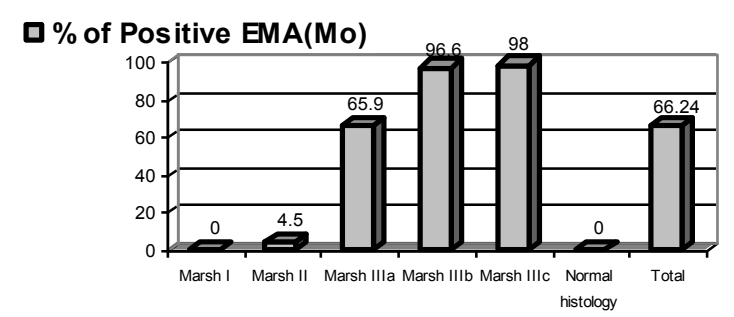

Fig. 1- Bar chart showing the EMA (mo) positivity rates according to histopathological findings

EMA (uc): EMA by using human umbilical cord substrate; As shown in table 3 , there was a statistically significant positive trend between EMA (uc) positivity rate and severity of histological changes in coeliac patients. The rate increased from $70.7 \%$ for patients with Marsh IIla to $100 \%$ for those with Marsh IIIc, figure (2). Among children, a very high proportion $91 \%$ of coeliac patients were positive for EMA (uc) as compared to $0 \%$ for noncoeliac and control groups and this was highly significant statistically. The same was applicable for adults where the EMA(uc) positivity rate was $100 \%$ in coeliac group to $0 \%$ in non-coeliac and control groups.

Like in the EMA (mo), the EMA (uc) positivity rates for both gender groups were small in children and with no difference in adults, while the EMA (uc) positivity rate was $91 \%$ in children with coeliac compared to adults $100 \%$. As shown in table 4 , the PPV of EMA (uc) test in both children and adults was (100\%) for patients with a clinical suspicious of coeliac disease, but the NPV was $77.05 \%$ in children while it was $100 \%$ in adults. The sensitivity of EMA (uc) test among children was $93.38 \%$ while among adults $100 \%$. If this was to be used in screening for coeliac disease one can expect to find $93.30 \%$ of children and $100 \%$ of adults who have positive EMA (uc) are real coeliac patients, table 4.

Table 3- EMA (uc) positivity rates according to type and severity of histopathological findings

\begin{tabular}{|c|c|c|c|c|}
\hline \multirow[b]{2}{*}{ Histopathology } & \multicolumn{2}{|c|}{ Positive EMA (uc) } & \multirow[b]{2}{*}{ Total } & \multirow[b]{2}{*}{ P-value } \\
\hline & No. & $\%$ & & \\
\hline Marsh I & 0 & 0 & 29 & \multirow{7}{*}{0.00001} \\
\hline Marsh II & 0 & 0 & 22 & \\
\hline Marsh IIla & 29 & 70.7 & 41 & \\
\hline Marsh Illb & 85 & 97.7 & 87 & \\
\hline Marsh IIlc & 98 & 100 & 98 & \\
\hline Normal histology & 0 & 0 & 37 & \\
\hline Total & 212 & 67.52 & 314 & \\
\hline
\end{tabular}

Table 4- Performance characteristics of EMA (uc) test (Sensitivity and Specificity).

\begin{tabular}{|llll|}
\hline Children $(n=202)$ & EMA (uc) test & Coeliac & Non-coeliac \\
Sensitivity $=93.38 \%$ & Positive & 141 & 0 \\
Specificity $=100 \%$ & Negative & 14 & 47 \\
PPV $=100 \%$ NPV $=$ & Total & 155 & 47 \\
$77.05 \%$ & EMA (uc)test & Coeliac & Non-coeliac \\
Adults $(n=112)$ & Positive & 71 & 0 \\
Sensitivity $=100 \%$ & Negative & 0 & 41 \\
Specificity $=100 \%$ & Total & 71 & 41 \\
PPV $=100 \%$ NPV $=$ & & & \\
$100 \%$ & &
\end{tabular}




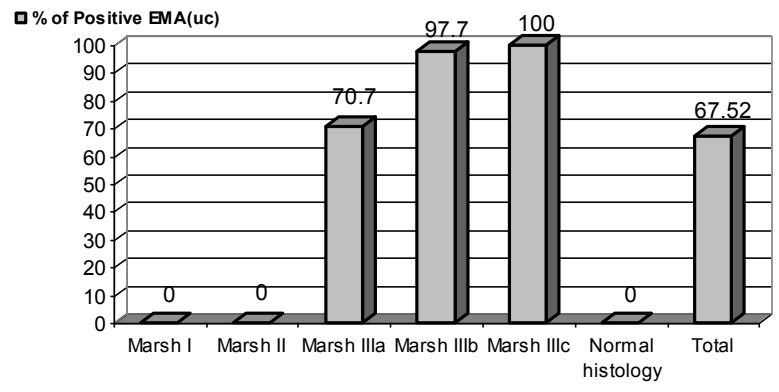

Fig. 2- Bar chart showing the EMA (uc) positivity rates according to type and severity of histopathological findings.

\section{Discussion}

According to our knowledge this comparison study by using two substrates of EMA was performed for the first time in Iraq, however in this country, $C D$ may be is one of the most prevalent life-long gastrointestinal diseases. [13-15] In untreated CD, ingested gluten triggers the production of endomysial antibodies, IgA class in the serum and tissue. [16] The most effective test for the diagnosis of active $C D$ is the assessment of antiendomysium antibodies beside anti-tissue transglutaminase.[17]

In this work home made substrate using human umbilical cord was employed for testing EMA to compare the results with commercial kit in which monkey esophagus was used as a substrate.

The study showed a correlation between the severity of intestinal mucosal damage of $C D$ and the appearance of EMA in both substrates. We found that the specific honey comb binding of EMA to the connective tissue surrounding muscle cells of vessels within the umbilical cord in all tested coeliac patients with histological finding of $C D$ that is (Marsh IIIc). This was the same finding of many workers. [18- 20] However binding was absent in patients with Marsh I, Marsh II, non-coeliac patients and normal subjects. Further more. In 5 of our untreated coeliac disease cases EMA bound only to human tissue umbilical cord but not to monkey esophagus, this confirming that human tissue is a more sensitive material than that of animals.

Partial villous atrophy seemed to be a quite frequent histopathological presentation in our coeliac population (41/226) $18.1 \%$. The question is how many coeliac disease patients will be missed if we do screening programs that rely too much on serology. Interestingly, partial villous atrophy and negative serology coexist with a co-occurrence of approximately $(29.2 \%, 12 / 41$ of cases in this study). Therefore, the predictive value of serology in coeliac patients with Marsh Illa appears to be disappointing. Our results confirm the suggestions in the pediatric literatures in which patients with detectable EMA at the time of biopsy exhibited more advanced villous atrophy. Data related to the value of EMA in untreated coeliac patients with Marsh IIla are lacking in the literatures. [21 - 23] There is a further consideration which needs attention. At present, there is no discussion in the literatures about EMA-negative coeliac patients. It is important to avoid a selffulfilling prophecy, taking biopsies only from EMA-positive individuals. Small bowel biopsy should be the first diagnostic procedure when there is a clinical suspicion of coeliac disease, in spite of positive or negative results of serology.

For the IgA- EMA test, the majority of studies report sensitivity in excess of $95 \%$. In our study, the sensitivity and the specificity stand respectively at $96.6 \%$ and $100 \%$ on human umbilical cord in the total number of children and adults. The percentages may be slightly different from that found in the other countries might be related to the difference in the volume of samples or the sensitivity of kits or the difference in the stage of disease. But the higher sensitivity and specificity of EMA in Iraqi coeliac disease patients on human umbilical cord are an important step to use this substrate in local laboratories as non invasive and highly precise diagnostic tool for coeliac disease, this was consistent with many studies.[10, 24-27] Furthermore, this simple immunohistochemical method permits unlimited testing and characterization of EMA in an easily and commonly available and inexpensive human tissue, which is a perfectly adequate substitute than the distal oesophagus of animals (monkey) since monkey's esophagus is ethically questionable for large scale investigations. [18, 28, 29] .

\section{Conclusions}

Detecting the presence of serum antibodies (IgA EMA) was almost diagnostic for clinically suspected coeliac disease in children and adults. The use of human umbilical cord as a substrate (for the first time in Iraq) is ethical. Serological tests can be of help to identify $\mathrm{CD}$ in primary care and suitable as a screening tool since its sensitivity is very high $96.6 \%$, as they are not an invasive method; its cost is acceptable and cause very little discomfort.

\section{Conflict of interests}

The authors declare that there is no personal and funding conflict of interests associated with work.

\section{Authors' contribution}

We declare that work was done by all the authors named in this article and all the liabilities pertaining to claims relating to the content of this article will be borne by the authors. Muhamed T Osman coordinated the study design and participated in the all laboratory work and data collection and analysis and drafted the manuscript. Ghada Al Duboni and Balsam I Taha participated in the serological work. Sanaa A Al-Nasiry participated in the histopathology \& serology work and data analysis. Makki H Fayadh participated in doing the patient's endoscopy and clinical work. All the authors read the final manuscript.

\section{Acknowledgement}

The authors would like to thank staff of Histopathology and Immunology units at Teaching Laboratories of Medical City in Baghdad for providing materials used in this work and technical support..

\section{References}

[1] Green P.H. and Cellier C. (2007) N Engl J Med, 357, 1731-43.

[2] Dennis C.W.P., Huib C., Rob B.D. and Cornelis B. (2006) World J Gastroenterol, 7, 12(17), 2779-2780.

[3] Garampazzi A., Rapa A. and Mura S. (2007) Journal of Pediatric Gastroenterology and Nutrition, 45, 611-4.

[4] Peter H.R., Green K.R. and Michael N.M. (2005) Best practice and Research clinical Gastroenterology, 19(3), 389-400.

[5] Shamir R., Lerner A., Shinar E., Lahat N., Sobel E., Bar-or R., Kerner H. and Eliakim R. (2002) Am J Gastroenterol, 97, 2589 $-2594$.

[6] Abrams J.A., Diamond B., Rotterdam H. and Green P.H.R. 
(2004) Atrophy. Dig Dis Sci, 49, 546-550.

[7] Salmi T.T., Collin P., Korponay-Szabo I.R., Laurila K., Partanen J. and Huhtala H. (2006) Gut, 55, 1746-1753.

[8] Fowell A.J., Thomas P.W., Surgenor S.L. and Snook J.A. (2006) Q J Med, 99, 453-460.

[9] Chorzelski T.P., Beutner E.H. and Sulej J. (1984) Br J Dermatol, 111, 395-9.

[10]Ladinser B., Rossipal E. and Pittschieler K. (1994) Gut, 35, 776-8.

[11]Marsh M.N. (1992) Gastroenterology, 102, 330-354.

[12] Oberhuber G., Granditsch G. and Vogelsang H. (1999) Eur J Gastroenterol Hepatol, 11, 1185-1194.

[13]Sanna A.A. and Muhamed T.O. (2004) Journal Faculty of Medicine, Baghdad, 46, 10-14.

[14]Zuhair A.K., Taha A.M. and Hudda Z. (1996) J Faculty of Medicine, Baghdad, 38 (2), 116-122.

[15]Sarmad A.E. (2003) Iraqi College of medicine, Al-Nahrain University. Baghdad.

[16]Dahle C., Hagman A., Ignatova S., and Stro"m M. (2010) Aliment Pharmacol Ther, 32, 254-260.

[17]Hopper A.D., Hadjivassiliou M. and Hurlstone D.P. (2008) Clinical Gastroenterology and Hepatology, 6, 314-20.

[18]Brandimarte G., Tursi A. and Giorgetti G.M. (2002) Minerva Gastroenterologica Dietologica, 48, 121-30.

[19]Christopher P.C., Bogera P.W., Thomasc D., Nicholasb S., Surgenora S.L. and Jonathon A.S. (2007) European Journal of Gastroenterology \& Hepatology, 19, 890-895.

[20]Agardh D. (2007) Clinical Gastroenterology and Hepatology, 5, 1276-81.

[21]Corrao G., Corazza G.R. and Andereani M.L. (1994) Gut, 35, 771-775.

[22]Rampertab S.D., Pooran N. and Brar P. (2006) American Journal of Medicine, 119, 355-14.

[23]Yagil Y., Goldenberg I. and Arnon R. (2005) Digestive Diseases and Sciences, 50, 796-805.

[24]Dorn S.D. and Matchar D.B. (2008) Digestive Diseases and Sciences, 53, 680-8.

[25]Vilppula A., Collin P. and Maki M. (2008) Digestive and Liver Disease, 40, 809-13.

[26]McAdam S.N. and Sollid L.M. (2000) Gut, 47, 743-5.

[27]Atkinson K., Tokmakajian S., Watson W. and Gregor J. (1997) Can J Gastroenterol, 11, 673-7.

[28]Kolho K.L., Farkkila M.A. and Savilahti E. (1998) Scand J Gastroenterol, 33, 1280.

[29]Volta U., Molinaro M., Franceschi D.L., Fratangelo D. and Bianchi F.B. (1995) Dig Dis Sci, 40, 1902-5. 